\title{
Estimation and Categorization of Errors in Error Recovery Using Task Stratification and Error Classification
}

\author{
Akira Nakamura, Kazuyuki Nagata \\ Intelligent Systems Research Institute \\ National Institute of Advanced Industrial Science and Technology (AIST) \\ Central 2, 1-1-1 Umezono, Tsukuba, Ibaraki, 305-8568 Japan \\ Kensuke Harada \\ Robotic Manipulation Research Group \\ Systems Innovation Department \\ Graduate School of Engineering Science, Osaka University \\ 1-3 Machikaneyama, Toyonaka 560-8531, Japan \\ Natsuki Yamanobe \\ Intelligent Systems Research Institute \\ National Institute of Advanced Industrial Science and Technology (AIST) \\ Central 2, 1-1-1 Umezono, Tsukuba, Ibaraki, 305-8568 Japan \\ E-mail:a-nakamura@aist.go.jp,k-nagata@aist.go.jp, \\ harada@sys.es.osaka-u.ac.jp,n-yamanobe@aist.go.jp \\ www.aist.go.jp
}

\begin{abstract}
We proposed an approach to error recovery that uses the concepts of task stratification and error classification. In our method, errors are classified according to the estimated cause into several categories, such as modeling and planning errors. When an error is classified correctly, this increases the probability that the most suitable recovery is performed. In this paper, we describe a procedure for error categorization.
\end{abstract}

Keywords: error recovery, task stratification, error classification, manipulation, planning

\section{Introduction}

For robots that perform manipulation tasks for plant maintenance and industrial production, error recovery is an important research topic. ${ }^{1-4}$ However, systematic methods of error recovery have not yet been reported. We propose an approach to error recovery that uses the concepts of task stratification and error classification. 5,6 With our error recovery technique, a highly reliable system expression is derived by analyzing the error and estimating the cause, and the work process continues based on the expressions of the corrected system.
In error recovery, errors are judged as a process of system operation. In our method, errors that occur are classified according to the estimated cause into several categories: sensing, modeling, planning, and execution errors. When an error is classified correctly, this makes it more likely that the most suitable recovery is performed. In this paper, we describe an error categorization procedure. We show the procedure for two cases: with and without experience practicing manipulation tasks. Our methods are efficient because the system revisions start from a small scale in the case 
of no experience and from parts with a high probability of error occurrence in the case of experience.

The rest of this paper is organized as follows. Section 2 describes the concept of error recovery. Section 3 presents the error classification method, and Section 4 proposes the recovery process in which the classification is used. Section 5 concludes this paper.

\section{Concept of Error Recovery}

In this section, we describe the basis of the error recovery technique. The target works are robot applications in industrial factories and power generation facilities: assembly, resolution, and parts exchange. In recent years, robots have become an increasingly active presence in the lives of people; thus, domestic works such as cleaning, washing, and cooking are also targets of our error recovery system.

We used an error recovery technique based on the concepts of task stratification and error classification, which we described in Ref. 5 . We proposed a new type of error recovery method that uses both forward and backward passes in Ref. 6.

Figure 1 shows a diagram for the basic concept of our error recovery. The center of the robot work is the main part. It consists of elements with sensing, modeling, planning, and execution sequences. Our

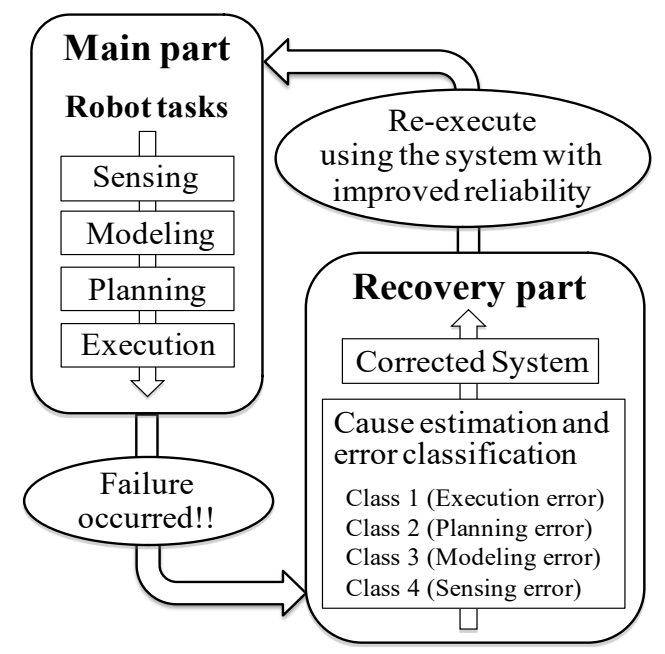

Fig. 1 Robot task system with an error recovery function method comprises a hierarchy from the main work objective to bottom layers with sensing, modeling, planning, and execution flows for each hierarchy.

If failure occurs in the main part, the process advances to the recovery part (Fig. 1). The basic flow in this part is as follows: the cause of the error is estimated, the error is classified, the system is revised, and re-execution is performed in the system for improved reliability.

\section{Cause of Error}

The work of the robot consists of sensing, modeling, planning, and operation flows. Therefore, four types of errors were considered and classified. For simplification, each error was assumed to have only one cause in this study.

\section{(Class 1) Execution Error}

This error occurs when there is a problem with the machine, and a task can usually be achieved without failure if the same operation is repeated many times. When there is a specific problem with a mechanism, the most typical cause of the error is a gear backlash. The clearance is increased by abrasion. There can be many causes, such as changes in the temperature or humidity, vibrations, bending, deterioration with age, and reduced lubricant. Errors should be identified by using experience and artificial intelligence.

\section{(Class 2) Planning Error}

This error occurs when the system in computer software does not accurately express the real system. The error is mostly caused by setting mistakes for thresholds and parameters during the planning stage. If these are corrected, the task should be successfully performed. A correct system expression can also be derived by specifying the part to be corrected based on error analysis.

\section{(Class 3) Modeling Error}

This error occurs when the geometric model used in the computer software does not express the real object exactly. Therefore, the task may be successfully performed if the model is revised and the task is carried out again. When the model oversimplifies a real object, the necessary step is to change the model to represent the object more exactly. A model appropriate for 
performing a task more accurately can be derived by iteration.

\section{(Class 4) Sensing Error}

This error occurs when there are mistakes with sensing and insufficient calibration. When this is corrected, re-execution should allow a task to be successfully performed. This can generally be corrected by adjusting the camera parameters. In many cases, the operation of a task needs to be stopped for modifications.

The modification is carried out according to the classified error, and the task is performed again by using the system with improved reliability, as shown by the

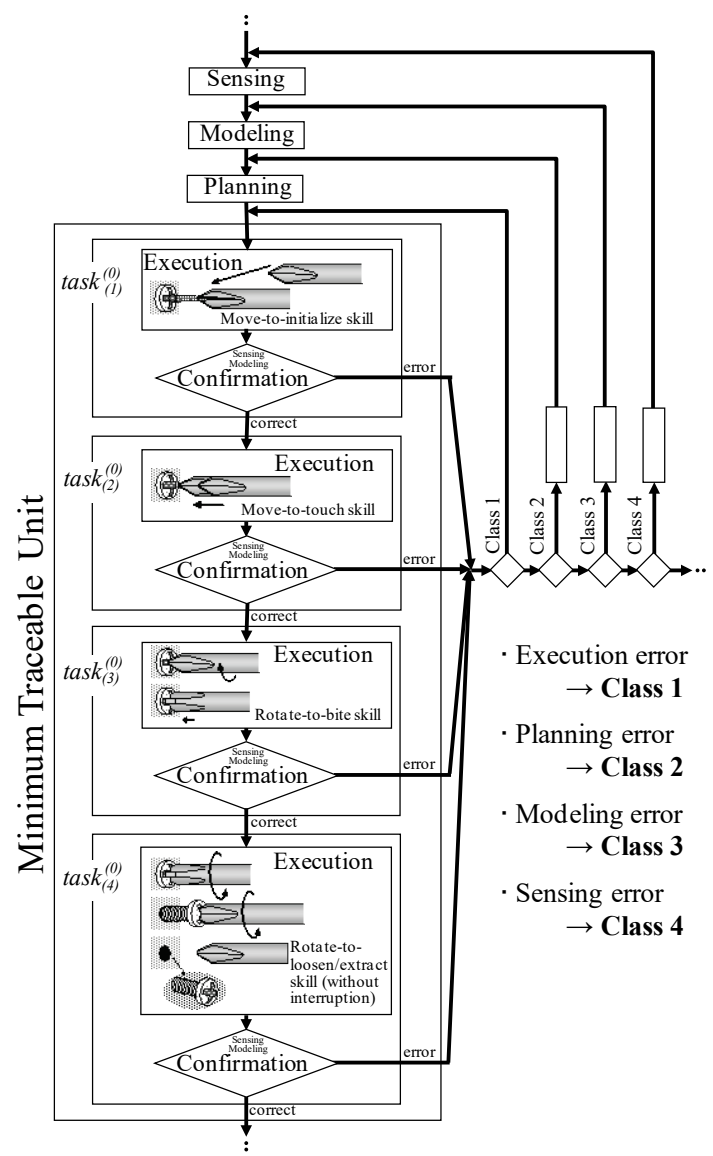

Fig. 2 Process flow with error recovery in skill sequence of loosening a screw using a screwdriver flowchart in Fig. 2. The task of loosening a screw with a screwdriver is used as an example.

\section{Error Recovery Process}

In this section, we consider how to take passes based on a probability of an error classification.

The error classification part in the system flow influences the ability of an operation to recover from an error (Fig. 3). The order of decision blocks when classifying errors in the process flow is especially important to this ability. If the cause of an error can be estimated to some extent, the error classification efficiency can be improved. We considered how to order decision blocks for the error classification box in the process flow according to the estimated error cause. If the cause of an error cannot be estimated with no experience, the system is revised by starting at a small scale. Here, we explain the error classification procedures for the cases of no experience and some experience.

(Case 1) Cause of an error cannot be estimated

By regarding an error cause as a perfectly virtual one, a recovery is performed. In this case, based on the flowchart for the recovery process as shown in Fig. 3, the cause is first regarded as an execution error (i.e.,

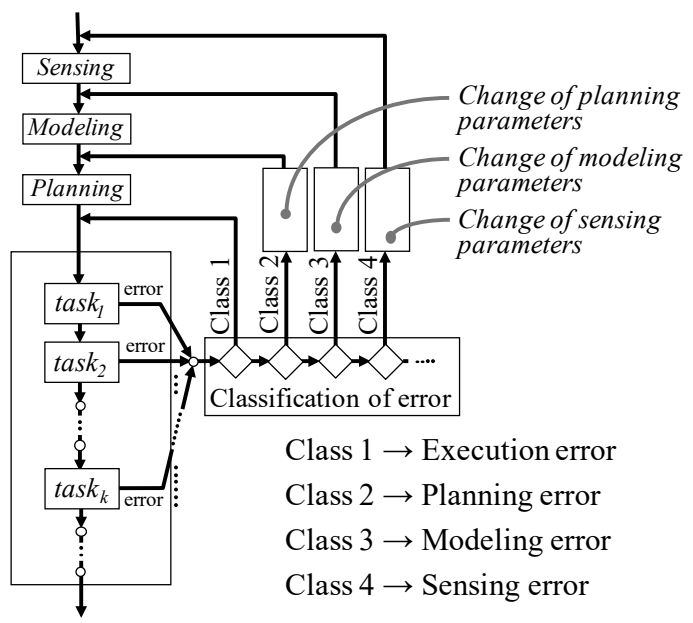

Fig. 3 Fundamental process flow with error recovery 
Class 1), and the task is merely repeated. When the error status continues even if the task is repeated more than one time, the cause is next regarded as a planning error (i.e., Class 2). If the numerical values of various parameters are changed, this may produce an improvement. Once an improvement is found, the task is carried out again, and the error status is checked. If the status has returned to normal, the cause can be assumed to be a planning error. If the task is not successfully performed, the Class 3 and 4 errors are checked successively through similar procedures. This method is efficient because the system is improved starting from a small scale.

(Case 2) Cause of an error can be estimated to some extent

In this case, the potential causes can be narrowed to some extent from experience and chosen from a list. The error causes are sorted by the order that they tend to occur, which changes the flowchart for the recovery (Fig. 3). For example, consider the following order of priority for error causes:

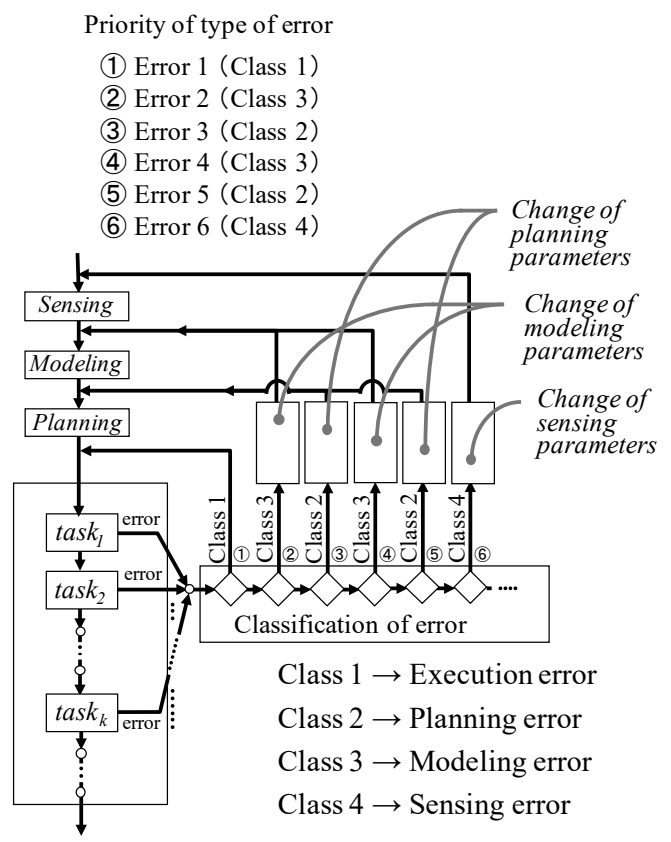

Fig. 4 Corrected process flow with error recovery (i) Error 1

Error type: (Class 1) Execution error due to gear backlash

Probability of occurrence: $p_{1}$

(ii) Error 2

Error type: (Class 3) Modeling error for a grasped object

Probability of occurrence: $p_{2}$

(iii) Error 3

Error type: (Class 2) Planning error in which control system parameters are inaccurate

Probability of occurrence: $p_{3}$

(iv) Error 4

Error type: (Class 3) Modeling error for the target object of an operation

Probability of occurrence: $p_{4}$

(v) Error 5

Error type: (Class 2) Planning error in which the threshold values for passing conditions are incorrect

Probability of occurrence: $p_{5}$

(vi) Error 6

Error type: (Class 4) Sensing error derived from insufficient calibration

Probability of occurrence: $p_{6}$

Fig. 4 shows the recovery flowchart when $p_{1}>p_{2}>p_{3}>$ $p_{4}>p_{5}>p_{6}$. This is an example derived from a case where $p_{1}=30 \%, p_{2}=25 \%, p_{3}=20 \%, p_{4}=12 \%, p_{5}=$ $8 \%$ and $p_{6}=5 \%$. The decision blocks for the error classification are ordered from high to low probability, which produces an efficient flowchart. The cause is specified according to the error situation, the task when an error occurs, and the process to correct it. Once the system is corrected, the task is re-executed with improved reliability.

\section{Conclusion}

We showed a way to classify the cause of an error and the recovery process for different cases. The estimation accuracy for the error cause depends on the degree of experience. We divided the procedure into two cases: 
with and without experience. In addition, we described a flowchart to converge to the true error cause by going through each category. When the cause can be accurately determined, the system can operate correctly. This reduces the probability of an error in later tasks. Future studies will involve applying our method to an actual system and search for a method that can derive the cause of an error more exactly even with little experience.

\section{Acknowledgement}

This work was supported by JSPS KAKENHI Grant Number 15K00370.

\section{References}

1. B. R. Donald, Planning multi-step error detection and recovery strategies, Int. J. Robot. Res., 9(1) (1990) 3-60.
2. E. Z. Evans and C. S. George Lee, Automatic generation of error recovery knowledge through learned reactivity, in Proc. IEEE Int. Conf. Robot. Autom., (San Diego, USA, 1994), 2915-2920.

3. C. M. Baydar and K. Saitou, Off-Line error prediction, diagnosis and error recovery using virtual assembly systems, in Proc. IEEE Int. Conf. Robot. Autom., (Seoul, Korea, 2001), 818-823.

4. C. W. Moon and B. H. Lee, A model-based error recovery scheme for a multi-robot system, Robotica, Cambridge Univ. Press, 19(4) (2001) 371-380.

5. A. Nakamura and T. Kotoku, Systematization of error recovery in skill-based manipulation, Artificial Life and Robotics, Springer, 14(2) (2009) 203-208.

6. A. Nakamura, K. Nagata, K. Harada, N. Yamanobe, T. Tsuji, T. Foissotte and Y. Kawai, Error recovery using task stratification and error classification for manipulation robots in various fields, in Proc IEEE/RSJ Int. Conf. on Intell. Robots Syst., (Tokyo, Japan, 2013), 3535-3542. 